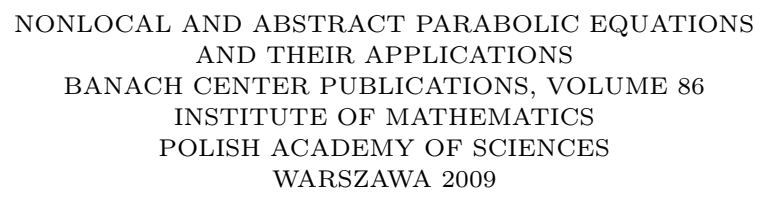

\title{
THE DIRICHLET PROBLEM IN WEIGHTED SPACES ON A DIHEDRAL DOMAIN
}

\author{
ADAM KUBICA \\ Faculty of Mathematics and Information Science \\ Warsaw University of Technology \\ Pl. Politechniki 1, 00-661 Warszawa, Poland, and \\ Institute of Mathematics and Cryptology \\ Military University of Technology \\ Kaliskiego 2, 00-908 Warszawa, Poland \\ E-mail: A.Kubica@mini.pw.edu.pl
}

\begin{abstract}
We examine the Dirichlet problem for the Poisson equation and the heat equation in weighted spaces of Kondrat'ev's type on a dihedral domain. The weight is a power of the distance from a distinguished axis and it depends on the order of the derivative. We also prove a priori estimates.
\end{abstract}

1. Introduction. In this paper we consider the Dirichlet problem for the Poisson equation and the heat equation in weighted spaces on a dihedral domain. The weight is a power of the distance from the $z$-axis and it depends on the order of the derivatives. This kind of problems were examined in many books and articles (see, for instance, [3], [7], [9], [10] and references therein). As far as the Dirichlet problem for the Poisson equation in a dihedral domain are concerned, the results presented here (except for a priori estimates) may be found, for example, in [9] (see Prop. 11.1.4) or in [10]. However, the proofs given there are rather complicated. We show here that the existence result can be obtained from the Lax-Milgram theorem, which requires only a suitable bilinear form satisfying the ellipticity condition. We want to stress that the argument of this type works well in two or three dimensional domains, which may be bounded or unbounded. Furthermore, this idea can be applied to other problems, for example to the operator $L_{\sigma}=-\Delta+\sigma r^{-2}$ on $\dot{\mathbb{R}}^{3}$ for $\sigma \geq 0$ and gives the existence of solutions on the maximal interval $\mu \in(1-\sqrt{\sigma}, 1+\sqrt{\sigma})$ of weight. The results for problems in the weighted spaces,

2000 Mathematics Subject Classification: 35J05, 35K05, 35J25, 35B45.

Key words and phrases: Poisson equation, heat equation, weighted spaces, dihedral domain, Dirichlet problem, a priori estimates.

The paper is in final form and no version of it will be published elsewhere. 
which depend upon the Lax-Milgram theorem may be found in [6] and [7]. However (see example 3.4 of [6]), they get the existence of solutions only on a smaller interval than the maximal one. Applying the method presented here to the problem from example 3.4 of [6] we get the existence on the maximal interval.

Now, we briefly describe the well-known approach in deriving the basic result ${ }^{1}$ :

$$
\mathcal{A}_{\mu}^{\vartheta} \text { is an isomorphism } \Leftrightarrow \mu \in(1-\pi / \vartheta, 1+\pi / \vartheta) .
$$

Namely, the following steps ought to be taken: $(i) \mathcal{A}_{\mu}^{\vartheta}$ is an isomorphism $\Leftrightarrow \mathcal{B}_{\mu}^{\vartheta}$ is an isomorphism (thm. 8.2.1 of [10]), $($ ii $) \mathcal{B}_{\mu}^{\vartheta}$ is an isomorphism for $\mu \in\left(\beta_{1}, \beta_{2}\right)$ (thm. 1.3.18 of [9]), (iii) $\mathcal{B}_{\beta}^{\vartheta}$ is not an isomorphism for $\beta \notin\left(\beta_{1}, \beta_{2}\right)$ (prop. 8.2 .9 of [10]).

The structure of our proof is as follows: we begin with the existence result (thm. 3.7) and then we obtain an a priori estimate (thm. 4.2). Further, we need some technical statements. Namely, we get a counterpart of $(i)$ (see prop. 5.5 and 5.6) and a modification of (iii) (see. thm. 5.8). Finally, we get $\operatorname{dim} \mathcal{K}_{\mu}^{\vartheta}=\operatorname{dim} \mathcal{N}_{\mu^{\prime}}^{\vartheta}$, where $\mu^{\prime}=2-\mu$ (thm. 5.10). These results lead us to theorem 5.12, i.e. $\mathcal{K}_{\mu}^{\vartheta} \neq\{0\}$ for $\mu>1+\pi / \vartheta$. We notice that the modification of $(i)$ - $(i i i)$ allows us to deal with problems which cannot be solved using (i)-(iii) only, for instance, the Poisson equation in $\dot{\mathbb{R}}^{3}$.

We also consider the heat equation in a bounded domain $D_{\vartheta, 1}$. Here again we establish ellipticity of our bilinear form and then we apply the Galerkin method. It is well-known that this is the first step of the regularizer technique (see [8]), which leads us to the solvability of other problems in domains with edges.

2. Notation. If $N \geq 2$ and $x \in \mathbb{R}^{N}$, then $r=r(x)$ equals $\sqrt{x_{1}^{2}+x_{2}^{2}}$. Let $\vartheta \in(0,2 \pi)$ and $d_{\vartheta}=\left\{x \in \mathbb{R}^{2}: r>0, \varphi \in(0, \vartheta)\right\}, d_{\vartheta, 1}=\left\{x \in d_{\vartheta}: r<1\right\}$ where $(r, \varphi)$ are the polar coordinates in $\mathbb{R}^{2}$. We write $\gamma_{k}=\left\{x \in \mathbb{R}^{2}: r>0, \varphi=k \vartheta\right\}, \gamma_{k, 1}=\left\{x \in \gamma_{k}: r<1\right\}$ for $k=0,1, D_{\vartheta}=d_{\vartheta} \times \mathbb{R}, D_{\vartheta, 1}=d_{\vartheta, 1} \times(-1,1), \Gamma_{k}=\gamma_{k} \times \mathbb{R}, \Gamma_{k, 1}=\gamma_{k, 1} \times(-1,1)$. If $U \subseteq \mathbb{R}^{N}$, then $\dot{U}=\bar{U} \backslash\{x: r=0\}$ and $U^{T}=U \times(0, T)$. Let $\varphi=\varphi(r)$ be a nondecreasing smooth function such that $\operatorname{supp} \varphi \subseteq(1, \infty)$ and $\varphi(r)=1$ for $r \geq \sqrt{2}$. For $x$ in $\mathbb{R}^{N}$ we set

$$
\eta_{n}(x):=\varphi\left(2^{-n} r\right)-\varphi\left(2^{-n-1} r\right) \quad \text { for } n \in \mathbb{Z} .
$$

Then, the family of functions $\left\{\eta_{n}\right\}_{n \in \mathbb{Z}}$ has the following properties:

$$
\begin{gathered}
0 \leq \eta_{n}(x) \leq 1 \text { for } x \in \mathbb{R}^{N}, \quad \eta_{0}\left(2^{-n} x\right)=\eta_{n}(x), \\
\quad \operatorname{supp} \eta_{n} \subseteq S_{n}:=\left\{x \in \mathbb{R}^{3} ; 2^{n}<r<2^{n+\frac{3}{2}}\right\},
\end{gathered}
$$

$$
\operatorname{supp} \eta_{n} \cap \overline{D_{\vartheta}} \subseteq S_{n}^{\vartheta}:=\left\{x \in \overline{D_{\vartheta}} ; \quad 2^{n}<r<2^{n+\frac{3}{2}}\right\}, \quad s_{n}^{\vartheta}:=\left\{x \in \overline{d_{\vartheta}} ; 2^{n}<r<2^{n+\frac{3}{2}}\right\} \text {, }
$$

$$
\begin{array}{r}
\eta_{n}(x)=1 \text { for } 2^{n+\frac{1}{2}} \leq r \leq 2^{n+1}, \quad\left|\eta_{n}^{(k)}(x)\right| \leq 2^{-k n} c_{0} \text { for } n \in \mathbb{Z} \text { and } k \\
\sum_{n \in \mathbb{Z}} \eta_{n}(x) \equiv 1 \text { on } \mathbb{R}^{N}, \quad \operatorname{supp} \eta_{n} \cap \operatorname{supp} \eta_{k}=\emptyset \Leftrightarrow|n-k|>1,
\end{array}
$$

where $c_{0}$ is a constant independent of $k$ and $n$. Furthermore, we need the following notation: $\eta_{\infty}:=\sum_{n=0}^{\infty} \eta_{n}, \bar{\eta}_{\infty}:=\eta_{\infty}-\eta_{0}$. We define $\chi_{n}(x):=\eta_{n-1}(x)+\eta_{n}(x)+\eta_{n+1}(x)$.

\footnotetext{
${ }^{1}$ See the next section for the notation.
} 
Then $\chi_{n} \cdot \eta_{n}=\eta_{n}, \operatorname{supp} \chi_{n} \subseteq\left\{x \in \mathbb{R}^{k}: r \in\left(2^{n-1}, 2^{n+\frac{5}{2}}\right)\right\}, \chi_{0}\left(2^{-n} x\right)=\chi_{n}(x)$, $\sum_{n \in \mathbb{Z}} \chi_{n}(x) \equiv 3$ for $x \in \dot{\mathbb{R}}^{N}, \operatorname{supp} \chi_{n} \cap \operatorname{supp} \chi_{k}=\emptyset \Leftrightarrow|n-k|>2$. We write $\chi_{\infty}:=\sum_{n=0}^{\infty} \chi_{n}, \quad \bar{\chi}_{\infty}:=\chi_{\infty}-\chi_{0}$. Now, we assume that $U \subseteq \mathbb{R}^{N}$ and we introduce the function spaces: if $f$ is defined on $\dot{U}$, then $f \in L_{2, \mu}(U) \Leftrightarrow\|f\|_{L_{2, \mu}(U)}=\left\|f \cdot r^{\mu}\right\|_{L^{2}(U)}<\infty$,

$$
\begin{aligned}
\mathcal{D}(\dot{U}) & =\left\{u \in C^{\infty}(\bar{U}): \operatorname{supp} u \text { is a compact subset of } \dot{U}\right\}, \\
H_{\text {loc }}^{m}(\dot{U}) & =\left\{u: u \in H^{m}(K) \text { for each compact subset } K \text { of } \dot{U}\right\} .
\end{aligned}
$$

The space $H_{\mu}^{m}(U)\left(\operatorname{resp} . H_{\mu}^{2 m, m}\left(U^{T}\right)\right)$ is the completion of the set $\mathcal{D}(\dot{U})\left(\operatorname{resp} . \mathcal{D}\left(\dot{U}^{T}\right)\right)$ with respect to the norm

$$
\begin{aligned}
\|u\|_{H_{\mu}^{m}(U)} & =\left\{\sum_{|\alpha| \leq m}\left\|D^{\alpha} u\right\|_{L_{2, \mu-m+|\alpha|}(U)}^{2}\right\}^{\frac{1}{2}}, \\
\text { resp. } \quad\|u\|_{H_{\mu}^{2 m, m}\left(U^{T}\right)} & =\left\{\sum_{|\alpha|+2 s \leq 2 m}\left\|D_{t}^{s} D^{\alpha} u\right\|_{L_{2, \mu-2 m+|\alpha|+2 s}\left(U^{T}\right)}^{2}\right\}^{\frac{1}{2}} .
\end{aligned}
$$

If $V$ is a space of functions defined on $U$ (resp. $U^{T}$ ), then $\stackrel{\circ}{V}$ denotes the subspace of $V$, which consists of functions $u$ such that $u_{\mid \partial U}=0$ (resp. $u(\cdot, t)_{\mid \partial U}=0$ for $t \in(0, T)$ ). If $V$ is a space of functions defined on $U^{T}$, then $V$ is the subspace of $V$ defined by the condition $u_{\mid t=0}=0$. By $W_{2, \mu}^{m}(U)$ we denote the space of functions $u \in L_{2, \mu}(U)$ such that $D^{\alpha} u \in L_{2, \mu}(U)$ for $|\alpha| \leq m$. The norm is given by the formula $\|u\|_{W_{2, \mu}^{m}(U)}:=$ $\left\{\sum_{|\alpha| \leq m}\left\|D^{\alpha} u\right\|_{L_{2, \mu}(U)}^{2}\right\}^{\frac{1}{2}}$. Finally, we define the function space $E_{\mu}^{m}(U)$ as the completion of $\mathcal{D}(\dot{U})$ with respect to the norm $\|w\|_{E_{\mu}^{m}(U)}:=\left\{\sum_{k=0}^{m}\|w\|_{H_{\mu}^{k}(U)}^{2}\right\}^{1 / 2}$.

We define $\mathcal{A}_{\mu}^{\vartheta}$ to be the Laplace operator on $\stackrel{\circ}{H}_{\mu}^{2}\left(D_{\vartheta}\right)$, i.e. $\mathcal{A}_{\mu}^{\vartheta}: \stackrel{\circ}{H}_{\mu}^{2}\left(D_{\vartheta}\right) \rightarrow L_{2, \mu}\left(D_{\vartheta}\right)$, $\mathcal{A}_{\mu}^{\vartheta} u=\Delta u$, and $\mathcal{B}_{\mu}^{\vartheta}$ to be $\Delta-1$ on $\stackrel{\circ}{E}_{\mu}^{2}\left(d_{\vartheta}\right)$, i.e. $\mathcal{B}_{\mu}^{\vartheta}: \stackrel{\circ}{E}_{\mu}^{2}\left(d_{\vartheta}\right) \rightarrow L_{2, \mu}\left(d_{\vartheta}\right), \mathcal{B}_{\mu}^{\vartheta} u=(\Delta-1) u$. We consider the kernel and the co-kernel of $\mathcal{A}_{\mu}^{\vartheta}$,

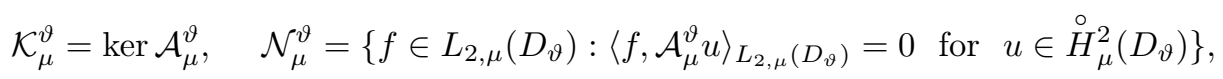

where $\langle\cdot, \cdot\rangle_{L_{2, \mu}\left(D_{\vartheta}\right)}$ denotes the inner product in $L_{2, \mu}\left(D_{\vartheta}\right)$. Furthermore, we set

$$
\mu^{\prime}:=2-\mu \text {. }
$$

Let $Q_{1}:=\mathbb{R}$ and $Q_{2}:=\mathbb{R}^{2}$ and let $s=s(q)$ be a function defined on $Q_{1}$ or $Q_{2}$ such that $s(q)=q^{2}$ for $q \in Q_{1}$ and $s(q)=q_{1}^{2}+i q_{2}$ for $q=\left(q_{1}, q_{2}\right) \in Q_{2}$.

We consider the following problems: the Poisson equation

$$
\left\{\begin{aligned}
\Delta u=f & \text { in } \quad D_{\vartheta}, \\
u=0 & \text { on } \quad \Gamma_{k}, \quad k=0,1 .
\end{aligned}\right.
$$

and the heat equation

$$
\left\{\begin{array}{rll}
u_{t}-\Delta u=f & \text { in } & D_{\vartheta, 1}^{T}, \\
u=0 & \text { on } & \Gamma_{k, 1}^{T}, \quad k=0,1 \\
u_{\mid t=0}=0 & \text { on } & D_{\vartheta, 1} .
\end{array}\right.
$$


Concerning the problem (E) we prove the following result:

Theorem 2.1. Assume that $\mu \neq 1+\pi \frac{k}{\vartheta}$ for $k \in \mathbb{Z} \backslash\{0\}$. Then

$$
\begin{gathered}
\exists c>0 \quad \forall u \in \stackrel{\circ}{H}_{\mu}^{2}\left(D_{\vartheta}\right) \quad\|u\|_{H_{\mu}^{2}\left(D_{\vartheta}\right)} \leq c\left\|\mathcal{A}_{\mu}^{\vartheta} u\right\|_{L_{2, \mu}\left(D_{\vartheta}\right)} \Leftrightarrow \mu<1+\frac{\pi}{\vartheta}, \\
\operatorname{dim} \mathcal{K}_{\mu}^{\vartheta}=\left\{\begin{array}{lll}
0 & \text { for } & \mu<1+\frac{\pi}{\vartheta}, \\
\infty & \text { for } & \mu>1+\frac{\pi}{\vartheta},
\end{array} \operatorname{dim}_{\mathcal{N}_{\mu}^{\vartheta}}=\left\{\begin{array}{lll}
\infty & \text { for } & \mu<1-\frac{\pi}{\vartheta}, \\
0 & \text { for } & \mu>1-\frac{\pi}{\vartheta},
\end{array}\right.\right. \\
\mathcal{A}_{\mu}^{\vartheta} \text { is an isomorphism } \Leftrightarrow \mu \in(1-\pi / \vartheta, 1+\pi / \vartheta) .
\end{gathered}
$$

Parts (9) and (10) are well-known, see for example proposition 11.1.4 and remark 11.1.5 of [9]. The above theorem is a consequence of theorems 3.7, 4.2 and corollary 5.14. The result concerning problem $(\mathrm{P})$ is as follows:

Theorem 2.2. Assume that $\mu \neq 1-\pi \frac{k}{\vartheta}$ for $k=1,2, \ldots$, and $T>0$. Then for $\mu<1+\frac{\pi}{\vartheta}$ we have

$$
\|u\|_{H_{\mu}^{2,1}\left(D_{\vartheta, 1}^{T}\right)} \leq c\left\|u_{t}-\Delta u\right\|_{L_{2, \mu}\left(D_{\vartheta, 1}^{T}\right)} \quad u \in{\stackrel{\circ}{H_{\circ}}}_{\mu}^{2,1}\left(D_{\vartheta, 1}^{T}\right),
$$

where $c=c(\mu, \vartheta)$. Furthermore, if $\mu \in\left(1-\frac{\pi}{\vartheta}, 1+\frac{\pi}{\vartheta}\right)$, then for each $f \in L_{2, \mu}\left(D_{\vartheta, 1}^{T}\right)$ there exists a unique solution $u \in \stackrel{\circ}{H}_{\circ}^{2,1}\left(D_{\vartheta, 1}^{T}\right)$ of $(P)$.

3. Existence of solutions. First, we recall the well known propositions concerning weighted spaces. Using the properties of functions $\eta_{n}$ and $\chi_{n}$ one can prove the following proposition.

Proposition 3.1. Assume that $\mu \in \mathbb{R}$. Then, there exists a constant $c=c(\mu)$ such that for $p:=\eta$ or $p:=\chi$ the following estimates hold:

$$
\begin{aligned}
c^{-1}\|u\|_{X} \leq & \left(\sum_{n=-\infty}^{\infty}\left\|p_{n} u\right\|_{X}^{2}\right)^{\frac{1}{2}} \leq c\|u\|_{X} \quad \text { for } u \in X, X=H_{\mu}^{2}\left(d_{\vartheta}\right), H_{\mu}^{2}\left(D_{\vartheta}\right), \\
& \sum_{n=1}^{\infty}\left\|p_{n} u\right\|_{X}^{2} \leq c\left\|p_{\infty} u\right\|_{X}^{2} \quad \text { for } u \in X, X=W_{2, \mu}^{1}\left(d_{\vartheta}\right), E_{\mu}^{2}\left(d_{\vartheta}\right) .
\end{aligned}
$$

Proposition 3.2. Assume that $u \in \stackrel{\circ}{\mathcal{D}}\left(\stackrel{\bullet}{D}_{\vartheta}\right)$. Then $\left\|D^{2} u\right\|_{L^{2}\left(D_{\vartheta}\right)}=\|\Delta u\|_{L^{2}\left(D_{\vartheta}\right)}$.

Proof. Suppose that $u \in \stackrel{\circ}{\mathcal{D}}\left(\dot{D}_{\vartheta}\right)$. It is sufficient to show that

$$
\int_{D_{\vartheta}} u_{x x} u_{y y} d x d y d z=\int_{D_{\vartheta}}\left|u_{x y}\right|^{2} d x d y d z, \quad \int_{D_{\vartheta}} u_{s s} u_{z z} d x d y d z=\int_{D_{\vartheta}}\left|u_{s z}\right|^{2} d x d y d z,
$$

where $s=x$ or $s=y$. We begin with the first equality in (14). Integrating by parts twice we obtain

$$
\int_{D_{\vartheta}} u_{x x} u_{y y} d x d y d z=\int_{D_{\vartheta}}\left|u_{x y}\right|^{2} d x d y d z-\sum_{k=0}^{1} \int_{\Gamma_{k}} u_{x} \partial_{\tau_{1}} u_{y} d \sigma,
$$

where $n=\left(n_{x}, n_{y}, n_{z}\right)$ is the outward normal vector to the boundary, $\tau_{1}=\left(-n_{y}, n_{x}, 0\right)$ is a tangent vector and $\partial_{\tau_{1}} u_{y}$ denotes the derivative of $u_{y}$ in the direction of $\tau_{1}$. The function $u$ vanishes on $\Gamma_{k}$ and $\tau_{1}$ is a tangent vector to $\Gamma_{k}$, hence $\partial_{\tau_{1}} u=0$ on $\Gamma_{k}$, 
i.e. $-n_{y} u_{x}+n_{x} u_{y}=0$ on $\Gamma_{k}$. If $n_{x}=0$ on $\Gamma_{k}$, then $u_{x}=0$ on $\Gamma_{k}$ and the integral over $\Gamma_{k}$ vanishes. If $n_{x} \neq 0$ on $\Gamma_{k}$, then $u_{y}=\frac{n_{y}}{n_{x}} u_{x}$ on $\Gamma_{k}$, hence $\int_{\Gamma_{k}} u_{x} \partial_{\tau_{1}} u_{y} d \sigma=$ $\frac{1}{2} \frac{n_{y}}{n_{x}} \int_{\Gamma_{k}} \partial_{\tau_{1}} u_{x}^{2} d \sigma=0$, because $\operatorname{supp} u$ is a compact subset of $\dot{D}_{\vartheta}$. Therefore, we get the first equality in (14). To obtain the second one, we integrate by parts twice and we have

$$
\int_{D_{\vartheta}} u_{s s} u_{z z} d x d y d z=\int_{D_{\vartheta}}\left|u_{s z}\right|^{2} d x d y d z-\sum_{k=0}^{1} \int_{\Gamma_{k}} u_{z} \cdot u_{s z} \cdot n_{s}-u_{z} \cdot u_{s s} \cdot n_{z} d \sigma .
$$

The function $u$ vanishes on $\Gamma_{k}$ and $(0,0,1)$ is a tangent to $\Gamma_{k}$, hence $u_{z}=0$ on $\Gamma_{k}$. Therefore, the integrals over $\Gamma_{k}$ vanish and the proof is complete.

Analogously, we can prove

Proposition 3.3. Assume that $u \in \stackrel{\circ}{\mathcal{D}}\left(\dot{d}_{\vartheta}\right)$. Then $\left\|D^{2} u\right\|_{L^{2}\left(d_{\vartheta}\right)}=\|\Delta u\|_{L^{2}\left(d_{\vartheta}\right)}$.

Using truncated functions and the convolution properties, we prove the following.

Proposition 3.4. Assume that $\mu$ is real. Then the set $\stackrel{\circ}{\mathcal{D}}\left(\dot{d}_{\vartheta}\right)$ is dense in $\stackrel{\circ}{H}_{\mu}^{2}\left(d_{\vartheta}\right)$ and $\stackrel{\circ}{E}_{\mu}^{2}\left(d_{\vartheta}\right)$ and the set $\stackrel{\circ}{\mathcal{D}}\left(\stackrel{\circ}{D}_{\vartheta}\right)$ is dense in $\stackrel{\circ}{H}_{\mu}^{2}\left(D_{\vartheta}\right)$.

Lemma 3.5. Assume that $\mu$ is real and $U=d_{\vartheta}, d_{\vartheta, 1}, D_{\vartheta}$ or $D_{\vartheta, 1}$. Then there exists a constant $c=c(\mu)$ such that for $u \in \stackrel{\circ}{H_{l o c}^{2}}(\dot{\bullet})$ the following estimate holds:

$$
\|u\|_{H_{\mu}^{2}(U)} \leq c\left\{\|\Delta u\|_{L_{2, \mu}(U)}+\|u\|_{L_{2, \mu-2}(U)}\right\} .
$$

REMARK 3.6. This lemma implies that if $u \in \stackrel{\circ}{H}_{l o c}^{2}(\dot{U})$ and the r.h.s. of $(15)$ is finite, then $u$ belongs to $H_{\mu}^{2}(U)$.

Proof. We show the estimate (15) only in the case of $U=D_{\vartheta}$, because in the remaining cases we proceed analogously. Furthermore, it is sufficient to show $(15)$ for $u \in \stackrel{\circ}{\mathcal{D}}\left(\dot{\circ}_{\vartheta}\right)$. First, using proposition 3.2 and the Hardy inequality ${ }^{2}$ we obtain

$$
\|\psi\|_{H_{\mu}^{2}\left(S_{n}\right)} \leq c\|\Delta \psi\|_{L_{2, \mu}\left(S_{n}^{\vartheta}\right)} \quad \text { for } \quad \psi \in \stackrel{\circ}{\mathcal{D}}\left(S_{n}^{\vartheta}\right), \quad n \in \mathbb{Z}
$$

where $c=c(\mu)$. If $u \in \stackrel{\circ}{\mathcal{D}}\left(\dot{\circ}_{\vartheta}\right)$, then we apply (16) with $u_{n}=\eta_{n} u \in \stackrel{\circ}{\mathcal{D}}\left(S_{n}^{\vartheta}\right)$ and we get $\left\|u_{n}\right\|_{H_{\mu}^{2}\left(D_{\vartheta}\right)}^{2} \leq c\left\{\|\Delta u\|_{L_{2, \mu}\left(S_{n}^{\vartheta}\right)}^{2}+\|u\|_{H_{\mu-1}^{1}\left(S_{n}^{\vartheta}\right)}^{2}\right\}$, where $c=c(\mu)$. Hence, adding these inequalities for $n \in \mathbb{Z}$ and utilizing (4) and (12) we obtain

$$
\|u\|_{H_{\mu}^{2}\left(D_{\vartheta}\right)} \leq c(\mu)\left\{\|\Delta u\|_{L_{2, \mu}\left(D_{\vartheta}\right)}+\|u\|_{H_{\mu-1}^{1}\left(D_{\vartheta}\right)}\right\} .
$$

On the other hand, after integrating by parts we have $\|\nabla u\|_{L_{2, \mu-1}\left(D_{\vartheta}\right)}^{2} \leq\|\Delta u\|_{L_{2, \mu}\left(D_{\vartheta}\right)}^{2}+$ $\left(4|\mu-1|^{2}+1\right)\|u\|_{L_{2, \mu-2}\left(D_{\vartheta}\right)}^{2}$. Thus, from (17) we get $(15)$ for $u \in \stackrel{\circ}{\mathcal{D}}\left(\dot{\bullet}_{\vartheta}\right)$.

Now, using the Lax-Milgram theorem, we prove the existence of solutions of the problem (E).

\footnotetext{
${ }^{2}$ In the case of $\mu=0$ and $\mu=1$ we cannot directly apply the Hardy inequality and we have to modify the power of $r$. Utilizing the assumption $\operatorname{supp} \psi \subseteq S_{n}^{\vartheta}$ we get the desired estimate.
} 
Theorem 3.7. Assume that $\mu \in\left(1-\frac{\pi}{\vartheta}, 1+\frac{\pi}{\vartheta}\right)$. Then for each $f \in L_{2, \mu}\left(D_{\vartheta}\right)$ there exists a unique solution $u \in H_{\mu}^{2}\left(D_{\vartheta}\right)$ of the problem $(D)$ and the following estimate holds:

$$
\|u\|_{H_{\mu}^{2}\left(D_{\vartheta}\right)} \leq c\|f\|_{L_{2, \mu}\left(D_{\vartheta}\right)},
$$

where $c$ depends only on $\vartheta$ and $\mu$.

Proof. We introduce the notation $H:=\left\{u \in H_{\mu-1}^{1}\left(D_{\vartheta}\right): u_{\mid \partial D_{\vartheta}}=0\right\}, L:=L_{2, \mu}\left(D_{\vartheta}\right)$ and $B[\cdot, \cdot]$ is a bilinear form defined on $H$ by

$$
B[u, \phi]=\int_{D_{\vartheta}} \nabla u \cdot \nabla \bar{\phi} \cdot r^{2 \mu-2} d x+(2 \mu-2) \int_{D_{\vartheta}} \nabla u \cdot \nabla r \cdot \bar{\phi} r^{2 \mu-3} d x .
$$

It is clear that $B[\cdot, \cdot]$ is continuous on $H$. We shall show that it is elliptic on $H$ if $|\mu-1|<\frac{\pi}{\vartheta}$. Indeed, direct calculations give $B[u, u]=\|\nabla u\|_{L_{2, \mu-1}\left(D_{\vartheta}\right)}^{2}-2|\mu-1|^{2}\|u\|_{L_{2 \mu-2}\left(D_{\vartheta}\right)}^{2}$. From the Hardy inequality (see th. 330 of [2]) we get $\left\|\frac{\partial u}{\partial r}\right\|_{L_{2, \mu-1}\left(D_{\vartheta}\right)}^{2}-|\mu-1|^{2}\|u\|_{L_{2, \mu-2}\left(D_{\vartheta}\right)}^{2} \geq 0$. Hence, if we denote by $\gamma$ the quantity $\frac{2\left(\frac{\vartheta}{\pi}\right)^{2}|\mu-1|^{2}}{1+\left(\frac{\vartheta}{\pi}\right)^{2}|\mu-1|^{2}}$, then

$$
\left\|\frac{\partial u}{\partial r}\right\|_{L_{2, \mu-1}\left(D_{\vartheta}\right)}^{2}-\gamma|\mu-1|^{2}\|u\|_{L_{2, \mu-2}\left(D_{\vartheta}\right)}^{2} \geq(1-\gamma)\left\|\frac{\partial u}{\partial r}\right\|_{L_{2, \mu-1}\left(D_{\vartheta}\right)}^{2}
$$

On the other hand, the Poincaré inequality yields $\|u\|_{L_{2, \mu-2}\left(D_{\vartheta}\right)} \leq \frac{\vartheta}{\pi}\left\|\frac{\partial u}{\partial \varphi}\right\|_{L_{2, \mu-2}\left(D_{\vartheta}\right)}$, hence

$$
\left\|\frac{\partial u}{\partial \varphi}\right\|_{L_{2, \mu-2}\left(D_{\vartheta}\right)}^{2}-(2-\gamma)|\mu-1|^{2}\|u\|_{L_{2, \mu-2}\left(D_{\vartheta}\right)}^{2} \geq(1-\gamma)\left\|\frac{\partial u}{\partial \varphi}\right\|_{L_{2, \mu-2}\left(D_{\vartheta}\right)}^{2} .
$$

Therefore estimates (19) and (20) give us $B[u, u] \geq(1-\gamma)\|\nabla u\|_{L_{2, \mu-1}\left(D_{\vartheta}\right)}^{2}$, where by the assumption on $\mu$ we have $1-\gamma>0$. Applying again the Hardy inequality and Poincaré inequality we obtain $B[u, u] \geq c\|u\|_{H_{\mu-1}^{1}\left(D_{\vartheta}\right)}^{2}$ for a positive constant $c=c(\vartheta, \mu)$. Therefore $B[\cdot, \cdot]$ is elliptic on $H$. Now, assume that $f$ belongs to $L_{2, \mu}\left(D_{\vartheta}\right)$. Then, from the Lax-Milgram theorem we get a unique $u \in H$ such that

$$
B[u, \phi]=\int_{D_{\vartheta}} f \bar{\phi} r^{2 \mu-2} d x \quad \forall \phi \in H, \quad\|u\|_{H} \leq c\|f\|_{L},
$$

where the constant $c$ depends only on $\vartheta$ and $\mu$. Using the standard argument, we deduce that $u \in \stackrel{\circ}{H}_{l o c}^{2}\left(\dot{D}_{\vartheta}\right)$ and $\Delta u=f$ in $D_{\vartheta}$. Thus, from lemma 3.5 and the estimate (21) we get that $u \in H_{\mu}^{2}\left(D_{\vartheta}\right)$. Hence, $u$ is a solution of (E) and the inequality (18) holds.

4. A priori estimates. First, we recall a well-known result concerning the problem in the angle $d_{\vartheta}$ (see [9] Theorem 1.2.1).

Theorem 4.1. Assume that $\mu \neq 1+\pi \frac{k}{\vartheta}$ for $k \in \mathbb{Z} \backslash\{0\}$. Then for each $f \in L_{2, \mu}\left(d_{\vartheta}\right)$ there exists a unique $u \in \stackrel{\circ}{H}_{\mu}^{2}\left(d_{\vartheta}\right)$ such that $\Delta u=f$ in $d_{\vartheta}$ and the estimate $\|u\|_{H_{\mu}^{2}\left(d_{\vartheta}\right)} \leq$ $c\|f\|_{L_{2, \mu}\left(d_{\vartheta}\right)}$ holds, where $c$ depends on $\mu$ and $\vartheta$.

In this section we obtain a priori estimates for the problem in the dihedral domain: 
THEOREM 4.2. Assume that $\mu<1+\frac{\pi}{\vartheta}$ and $\mu \neq 1-\pi \frac{k}{\vartheta}$ for $k=1,2, \ldots$ Then there exists a constant $c=c(\mu, \vartheta)$ such that

$$
\|u\|_{H_{\mu}^{2}\left(D_{\vartheta}\right)} \leq c\|\Delta u\|_{L_{2, \mu}\left(D_{\vartheta}\right)} \quad \text { for } \quad u \in \stackrel{\circ}{H}_{\mu}^{2}\left(D_{\vartheta}\right) .
$$

First, we prove the estimates for solutions of the elliptic problem in $d_{\vartheta}$ with a parameter.

REMARK 4.3. In the following lemma and its proof all differential operators depend upon $x \in \mathbb{R}^{2}$ variable and $q$ is a parameter. This Lemma is more general than we need here and it allows one to estimate the solutions of the heat equation in weighted spaces.

LEMmA 4.4. Assume that $\mu<1$ and $\mu \neq 1-\pi \frac{k}{\vartheta}$ for $k=1,2, \ldots$ Suppose that $i=1,2$ and $v$ is a smooth function on $d_{\vartheta} \times Q_{i}$. Assume that $v(\cdot, q) \in \stackrel{\circ}{\mathcal{D}}\left(\dot{\boldsymbol{d}}_{\vartheta}\right)$ for each $q \in Q_{i}$. Then there exists a constant $c=c(\mu, \vartheta)$ such that if

$$
g(x, q)=-\Delta v(x, q)+s v(x, q)
$$

then

$$
\sum_{k+l \leq 2} \int_{Q_{i}}|s|^{k}\left\|D^{2-k-l} v(\cdot, q)\right\|_{L_{2, \mu-l}\left(d_{\vartheta}\right)}^{2} d q \leq c \int_{Q_{i}}\|g(\cdot, q)\|_{L_{2, \mu}\left(d_{\vartheta}\right)}^{2} d q .
$$

Proof. In the case of $\mu \neq 0$ we repeat the proof of lemma 2 of [5], where the problem in $\mathbb{R}^{2}$ should be replaced by the problem in $d_{\vartheta}$ with the Dirichlet homogeneous boundary condition. Therefore, we only have to show (24) for $\mu=0$. In order to do it, we multiply both sides of $(23)$ by $(1-i \operatorname{Im} s)|s| \bar{v}$ and we integrate over $d_{\vartheta}$. After integrating by parts we get

$$
|s|^{1 / 2}\|D v(\cdot, q)\|_{L_{2,0}\left(d_{\vartheta}\right)}+|s|\|v(\cdot, q)\|_{L_{2,0}\left(d_{\vartheta}\right)} \leq 2\|g(\cdot, q)\|_{L_{2,0}\left(d_{\vartheta}\right)} \quad \text { for } \quad q \in Q_{i} .
$$

On the other hand, applying theorem 4.1 to the function $v(\cdot, q)$ and using $(23)$ we get

$$
\|v(\cdot, q)\|_{H_{0}^{2}\left(d_{\vartheta}\right)} \leq c\|g(\cdot, q)-s v(\cdot, q)\|_{L_{2,0}\left(d_{\vartheta}\right)} \quad \text { for } \quad q \in Q_{i},
$$

where $c=c(\vartheta)$. If we notice that the Schwarz inequality implies $|s|^{1 / 2}\|v(\cdot, q)\|_{L_{2,-1}\left(d_{\vartheta}\right)} \leq$ $|s|\|v(\cdot, q)\|_{L_{2,0}\left(d_{\vartheta}\right)}+\|v(\cdot, q)\|_{L_{2,-2}\left(d_{\vartheta}\right)}$, then (24) is a consequence of $(25)$ and $(26)$.

Proof of Theorem 4.2. In the case of $\mu \geq 1$ the estimate (22) is a consequence of theorem 3.7. Therefore, we have to consider the case of $\mu<1$. Due to proposition 3.4 it is sufficient to show

$$
\|u\|_{\stackrel{\circ}{H}_{\mu}^{2}\left(D_{\vartheta}\right)} \leq c\|\Delta u\|_{L_{2, \mu}\left(D_{\vartheta}\right)} \quad \text { for } \quad u \in \stackrel{\circ}{\mathcal{D}}\left(\dot{D}_{\vartheta}\right)
$$

with some $c=c(\mu, \vartheta)$. Thus, suppose that $u \in \stackrel{\circ}{\mathcal{D}}\left(\dot{D}_{\vartheta}\right)$ and denote by $v(x, q)$ the partial Fourier transform of $u(x, z)$ in the $z$ variable, i.e. $v(x, q):=\frac{1}{\sqrt{2 \pi}} \int_{-\infty}^{\infty} u(x, z) e^{-i z q} d z$. Let $f:=-\Delta u$ and $g(x, q):=\frac{1}{\sqrt{2 \pi}} \int_{-\infty}^{\infty} f(x, z) e^{-i z q} d z$. Then, the functions $v$ and $g$ satisfy the assumptions of lemma 4.4 in the case of $i=1$. Therefore, we get (24) and applying the Parseval identity to both sides of (24) we obtain (27). 
5. The relations between $\mathcal{A}_{\mu}^{\vartheta}$ and $\mathcal{B}_{\mu}^{\vartheta}$. The proof of the next statement is based on the proof of Lemma 8.2.4 of [10].

Proposition 5.1. Assume that $\mu$ is real. Then, there exists a constant $c=c(\mu)$ such that for $u \in \stackrel{\circ}{C}^{\infty}\left(\dot{d}_{\vartheta}\right)$ and $n \in \mathbb{N}$ the following estimate holds:

$$
\left\|\eta_{n} u\right\|_{E_{\mu}^{2}\left(d_{\vartheta}\right)}^{2} \leq c\left\{\left\|\eta_{n}(\Delta-1) u\right\|_{L_{2, \mu}\left(d_{\vartheta}\right)}^{2}+\left\|\chi_{n} u\right\|_{E_{\mu-1}^{1}\left(d_{\vartheta}\right)}^{2}\right\} .
$$

Proof. First, using proposition 3.3 and properties of $\eta_{0}$ and $\chi_{0}$ we show that

$$
\left\|\eta_{0} u\right\|_{H^{2}\left(d_{\vartheta}\right)} \leq c\left\{\left\|\eta_{0}(\Delta-1) u\right\|_{L_{2}\left(d_{\vartheta}\right)}+\left\|\chi_{0} u\right\|_{H^{1}\left(d_{\vartheta}\right)}\right\}
$$

for $u \in \stackrel{\circ}{C}^{\infty}\left(\dot{d}_{\vartheta}\right)$. Next, substituting $u_{n}(x):=u\left(2^{n} x\right)$ in (29) and changing the variables $y=2^{n} x$ we obtain

$$
\left\|\eta_{n} u\right\|_{H_{\mu}^{2}\left(d_{\vartheta}\right)}^{2} \leq c\left\{\left\|\eta_{n}(\Delta-1) u\right\|_{L_{2, \mu}\left(d_{\vartheta}\right)}^{2}+\left\|\chi_{n} u\right\|_{H_{\mu-1}^{1}\left(d_{\vartheta}\right)}^{2}+\left\|\eta_{n} u\right\|_{L_{2, \mu}\left(d_{\vartheta}\right)}^{2}\right\},
$$

for $u \in \stackrel{\circ}{C}^{\infty}\left(\dot{d}_{\vartheta}\right)$ and $n \in \mathbb{N}$ and where $c=c(\mu)$. On the other hand, integrating by parts gives us the inequality

$$
\left\|\eta_{n} u\right\|_{W_{2, \mu}^{1}\left(d_{\vartheta}\right)}^{2} \leq c\left\{\left\|\eta_{n}(\Delta-1) u\right\|_{L_{2, \mu}\left(d_{\vartheta}\right)}^{2}+\left\|\chi_{n} u\right\|_{L_{2, \mu-1}\left(d_{\vartheta}\right)}^{2}\right\},
$$

which holds for $u \in \stackrel{\circ}{C}^{\infty}\left(\dot{d}_{\vartheta}\right)$ and $n \in \mathbb{N}$ and $c=c(\mu)$. Therefore, from estimates (30) and (31) we get (28).

From this proposition we get $^{3}$

Corollary 5.2. Assume that $\mu<\beta$ and $v \in \stackrel{\circ}{C}^{\infty}\left(\dot{d}_{\vartheta}\right)$ is such that $(\Delta-1) v=0$ in $d_{\vartheta}$ and $\chi_{\infty} v \in E_{\mu}^{1}\left(d_{\vartheta}\right)$. Then $\bar{\eta}_{\infty} v \in E_{\beta}^{2}\left(d_{\vartheta}\right)$.

Proof. It is sufficient to consider the case $\beta=\mu+1$. If we apply proposition 5.1 with $v$ and $n=1,2, \ldots$ and use (13), then we get

$$
\frac{1}{3}\left\|\bar{\eta}_{\infty} v\right\|_{E_{\mu+1}^{2}\left(d_{\vartheta}\right)}^{2} \leq \sum_{n=1}^{\infty}\left\|\eta_{n} v\right\|_{E_{\mu+1}^{2}\left(d_{\vartheta}\right)}^{2} \leq c \sum_{n=1}^{\infty}\left\|\chi_{n} v\right\|_{E_{\mu}^{1}\left(d_{\vartheta}\right)}^{2} \leq \widetilde{c}\left\|\chi_{\infty} v\right\|_{E_{\mu}^{2}\left(d_{\vartheta}\right)}^{2}<\infty
$$

Assume that $x \in d_{\vartheta}, z \in \mathbb{R}$ and $u=u(x, z)$ belongs to $\mathcal{D}\left(\dot{D}_{\vartheta}\right)$. We define

$$
w(\eta, \xi):=\hat{u}(\eta /|\xi|, \xi) \quad \eta \in d_{\vartheta}, \quad \xi \in \dot{\mathbb{R}}
$$

where $\hat{u}(x, \xi):=\mathcal{F}_{z \mapsto \xi}[u(x, z)](\xi)$ stands for the partial Fourier transform with respect to the $z$ variable. Direct calculations give ${ }^{4}$

Proposition 5.3. If $u \in \mathcal{D}\left(\dot{D}_{\vartheta}\right)$ and $w$ is defined by formula (32), then

$$
\int_{\mathbb{R}}|\xi|^{2(1-\mu)}\|w(\cdot, \xi)\|_{E_{\mu}^{2}\left(d_{\vartheta}\right)}^{2} d \xi=\|u\|_{H_{\mu}^{2}\left(D_{\vartheta}\right)}^{2} .
$$

\footnotetext{
${ }^{3}$ See proposition 8.2 .6 of [10].

${ }^{4}$ See Lemma 8.1.2 of [10].
} 
COROLlary 5.4. Formula (32) defines an operator from $\mathcal{D}\left(\dot{D}_{\vartheta}\right)$ to $L_{2,1-\mu}\left(\mathbb{R} ; E_{\mu}^{2}\left(d_{\vartheta}\right)\right)$, which is linear and continuous with respect to the norm of $H_{\mu}^{2}\left(D_{\vartheta}\right)$. Therefore, it can be extended to the whole space $H_{\mu}^{2}\left(D_{\vartheta}\right)$ and then the equality (33) holds for each $u \in H_{\mu}^{2}\left(D_{\vartheta}\right)$. Furthermore, if $u \in \stackrel{\circ}{H}_{\mu}^{2}\left(D_{\vartheta}\right)$, then $w(\cdot, \xi) \in \stackrel{\circ}{E}_{\mu}^{2}\left(d_{\vartheta}\right)$ for a.e. $\xi \in \stackrel{\bullet}{\mathbb{R}}$.

Proposition 5.5. Assume that $\mu<1+\frac{\pi}{\vartheta}$ and $\mu \neq 1-\pi \frac{k}{\vartheta}$ for $k=1,2, \ldots$ Then there exists a constant $c=c(\mu, \vartheta)$ such that

$$
\|v\|_{E_{\mu}^{2}\left(d_{\vartheta}\right)} \leq c\|(\Delta-1) v\|_{L_{2, \mu}\left(d_{\vartheta}\right)} \quad \text { for } \quad v \in \stackrel{\circ}{E}_{\mu}^{2}\left(d_{\vartheta}\right) .
$$

Proof. Due to density of $\stackrel{\circ}{\mathcal{D}}\left(\dot{d}_{\vartheta}\right)$ in $\stackrel{\circ}{E}_{\mu}^{2}\left(d_{\vartheta}\right)$ it is enough to show $(34)$ for $v \in \stackrel{\circ}{\mathcal{D}}\left(\dot{d}_{\vartheta}\right)$. Let $\chi=\chi(t) \not \equiv 0$ be a smooth function with compact support and $\int_{\mathbb{R}}|\chi(t)|^{2} d t=1$ and set ${ }^{5}$

$$
u_{N}(x, z):=N^{-\frac{1}{2}} \exp (i z) \chi\left(z N^{-1}\right) v(x), \quad N \in \mathbb{N} .
$$

It is clear that $u_{N}$ are in $\stackrel{\circ}{H}_{\mu}^{2}\left(D_{\vartheta}\right)$, hence from theorem 4.2 we get $\left\|u_{N}\right\|_{H_{\mu}^{2}\left(D_{\vartheta}\right)} \leq$ $c\left\|\Delta u_{N}\right\|_{L_{2, \mu}\left(D_{\vartheta}\right)}$ for a constant $c=c(\mu, \vartheta)$. Then, taking the limit $N \rightarrow \infty$ we obtain (34) for $v \in \stackrel{\circ}{\mathcal{D}}\left(\dot{d}_{\vartheta}\right)$ with the same constant $c$.

Proposition 5.6. Assume that $\mu \leq 1$ and $\mu \neq 1-\pi \frac{k}{\vartheta}$ for $k=1,2, \ldots$ Suppose that the operator $\mathcal{A}_{\mu}^{\vartheta}$ is onto $L_{2, \mu}\left(D_{\vartheta}\right)$. Then the operator $\mathcal{B}_{\mu}^{\vartheta}$ is onto $L_{2, \mu}\left(d_{\vartheta}\right)$.

Proof. Let $\chi=\chi(\xi)$ be a smooth cut off function such that $0 \leq \chi \leq 1$ and $\chi(\xi)=1$ on $\left(\frac{1}{3}, \frac{2}{3}\right)$ and $\operatorname{supp} \chi \subseteq(0,1)$ and $\|\chi\|_{L^{2}(\mathbb{R})}=1$. For $g=g(\eta) \in \mathcal{D}(\dot{\dot{d}}, \vartheta)$ we define

$$
f_{g}(x, z):=\mathcal{F}_{\xi \mapsto z}^{-1}\left[|\xi|^{1+\mu} \chi(\xi) g(|\xi| x)\right](z), \quad x \in d_{\vartheta}, \quad z \in \mathbb{R} .
$$

Then $\left\|f_{g}\right\|_{L_{2, \mu}\left(D_{\vartheta}\right)}=\|g\|_{L_{2, \mu}\left(d_{\vartheta}\right)}$. Furthermore, the function $f_{g}$ is smooth in $\dot{D}_{\vartheta}$. By the assumptions and theorem 4.2 there exists a unique $u \in \stackrel{\circ}{H}_{\mu}^{2}\left(D_{\vartheta}\right)$ which satisfies $-\Delta u=f$. A standard argument implies that $u$ is smooth in $\dot{D}_{\vartheta}$. If we apply the partial Fourier transform $\mathcal{F}_{z \mapsto \xi}$ (denoted also by a hat $\left.{ }^{\wedge}\right)$, then we get

$$
\left(-\Delta_{x}+|\xi|^{2}\right) \hat{u}(x, \xi)=\hat{f}(x, \xi)=|\xi|^{1+\mu} \chi(\xi) g(|\xi| x) \quad \text { for } \quad x \in \dot{d}_{\vartheta}, \quad \xi \in \mathbb{R} .
$$

For $\xi \in \mathbb{R}$ we substitute $\eta:=|\xi| x$ and we obtain

$$
\left(-\Delta_{\eta}+1\right)\left[|\xi|^{1-\mu} \hat{u}\left(|\xi|^{-1} \eta, \xi\right)\right]=g(\eta) \quad \text { for } \quad \eta \in \dot{d}_{\vartheta}, \quad \xi \in(1 / 3,2 / 3) .
$$

The function $u$ belongs to $H_{\mu}^{2}\left(D_{\vartheta}\right)$, thus from proposition 5.3 we derive the inequality

$$
\int_{\mathbb{R}}|\xi|^{2(1-\mu)}\left\|\hat{u}\left(|\xi|^{-1} \cdot, \xi\right)\right\|_{E_{\mu}^{2}\left(d_{\vartheta}\right)}^{2} d \xi \leq\|u\|_{H_{\mu}^{2}\left(D_{\vartheta}\right)}^{2}<\infty .
$$

In particular $|\xi|^{1-\mu}\left\|\hat{u}\left(|\xi|^{-1} \cdot, \xi\right)\right\|_{E_{\mu}^{2}\left(d_{\vartheta}\right)}<\infty$ for $\xi \in\left(\frac{1}{2}, \frac{2}{3}\right)$. If we choose $\xi_{0} \in\left(\frac{1}{2}, \frac{2}{3}\right)$ and set $w(\eta):=\left|\xi_{0}\right|^{1-\mu} \hat{u}\left(\left|\xi_{0}\right|^{-1} \eta, \xi_{0}\right)$, then $w$ belongs to $\stackrel{\circ}{E}_{\mu}^{2}\left(d_{\vartheta}\right)$ and from (36) we get $(-\Delta+1) w=g$ in $\dot{d}_{\vartheta}$. Thus, for a given $g \in \mathcal{D}\left(\dot{d}_{\vartheta}\right)$ we defined $w \in \stackrel{\circ}{E}_{\mu}^{2}\left(d_{\vartheta}\right)$ such

\footnotetext{
${ }^{5}$ We use the idea which comes from the proof of theorem 8.2.1 of [10].
} 
that $(-\Delta+1) w=g$ in $\dot{d}_{\vartheta}$. From proposition 5.5 we have the estimate $\|w\|_{E_{\mu}^{2}\left(d_{\vartheta}\right)} \leq$ $c\|g\|_{L_{2, \mu}\left(d_{\vartheta}\right)}$. Therefore, we can extend this operator to the space $L_{2, \mu}\left(d_{\vartheta}\right)$.

We recall a useful proposition 8.2.7 from [10]:

Proposition 5.7. Assume that there is no number of the form $1+\pi \frac{k}{\vartheta}$ for $k \in \mathbb{Z} \backslash\{0\}$ between $\mu$ and $\gamma$. Then if $\mathcal{A}_{\mu}^{\vartheta}$ is an isomorphism, then $\mathcal{A}_{\gamma}^{\vartheta}$ is an isomorphism.

We use the following theorem instead of proposition 8.2.9 of [10].

TheOREM 5.8. Assume that $k \in \mathbb{N} \backslash\{0\}$ and $\mu, \beta$ satisfy

$$
1-\pi \frac{k+1}{\vartheta}<\mu<1-\pi \frac{k}{\vartheta}<\beta .
$$

If the operator $\mathcal{A}_{\mu}^{\vartheta}$ is onto $L_{2, \mu}\left(D_{\vartheta}\right)$, then the operator $\mathcal{A}_{\beta}^{\vartheta}$ is $\underline{\text { not }}$ injective.

Proof. Case of $1+k \frac{\pi}{\vartheta}+\mu>0$. For $x \in \dot{d}_{\vartheta}$ we set $\varsigma_{k}(x):=r^{\pi \frac{k}{\vartheta}} \sin \pi \frac{k}{\vartheta} \varphi$, where $r, \varphi$ are the polar coordinates in $\mathbb{R}^{2}$. Assume that $\eta=\eta(r)$ is a smooth cut off function, which is equal to 1 on $B_{1}\left(B_{r}\right.$ denotes the ball with center at the origin and radius $r$ ) and $\operatorname{supp} \eta \subseteq B_{2}$. Then $(\Delta-1)\left(\eta \varsigma_{k}\right)$ belongs to $L_{2, \mu}\left(d_{\vartheta}\right)$, because $1+k \frac{\pi}{\vartheta}+\mu>0$. By the assumption $\mathcal{A}_{\mu}^{\vartheta}$ is onto $L_{2, \mu}\left(D_{\vartheta}\right)$, hence from proposition 5.6 we deduce that $\mathcal{B}_{\mu}^{\vartheta}$ is onto $L_{2, \mu}\left(d_{\vartheta}\right)$. Thus, there exists $w \in \stackrel{\circ}{E}_{\mu}^{2}\left(d_{\vartheta}\right)$ such that $(\Delta-1) w=(\Delta-1)\left(\eta \varsigma_{k}\right)$ in $d_{\vartheta}$. We set

$$
v:=w-\eta \varsigma_{k} .
$$

First, we notice that $v \not \equiv 0$. Indeed, in the opposite case we would have $\eta \varsigma_{k}=w \in E_{\mu}^{2}\left(d_{\vartheta}\right)$ and then, in particular, $\eta \varsigma_{k} \in L_{2, \mu-2}\left(d_{\vartheta}\right)$, which is impossible since $\mu<1-\pi \frac{k}{\vartheta}$. Now, we shall show that $v$ belongs to $\stackrel{\circ}{E}_{\beta}^{2}\left(d_{\vartheta}\right)$. First, we write $v=\left(1-\bar{\eta}_{\infty}\right) v+\bar{\eta}_{\infty} v$. Clearly, for some $c$ we have $\left\|\left(1-\bar{\eta}_{\infty}\right) v\right\|_{E_{\beta}^{2}\left(d_{\vartheta}\right)} \leq c \sum_{l=0}^{2}\left\|D^{l} v\right\|_{L_{2, \beta-2+l}\left(B_{2^{3 / 2}}^{\vartheta}\right)}$. By definition of $v$ we have

$$
\left\|D^{l} v\right\|_{L_{2, \beta-2+l}\left(B_{2^{3 / 2}}^{\vartheta}\right)} \leq\left\|D^{l} w\right\|_{L_{2, \beta-2+l}\left(B_{2^{3 / 2}}^{\vartheta}\right)}+\left\|D^{l}\left(\eta \varsigma_{k}\right)\right\|_{L_{2, \beta-2+l}\left(B_{2^{3 / 2}}^{\vartheta}\right)} .
$$

By virtue of (37) we have $\mu<\beta$, thus for $l=0,1,2$ we get

$$
\left\|D^{l} w\right\|_{L_{2, \beta-2+l}\left(B_{2^{3 / 2}}^{\vartheta}\right)} \leq 2^{\frac{3}{2}(\beta-\mu)}\left\|D^{l} w\right\|_{L_{2, \mu-2+l}\left(B_{2^{3 / 2}}^{\vartheta}\right)}<\infty
$$

because $w \in E_{\mu}^{2}\left(d_{\vartheta}\right)$. By assumption we have $1-\pi \frac{k}{\vartheta}<\beta$, thus direct calculations give us $\left\|D^{l}\left(\eta \varsigma_{k}\right)\right\|_{L_{2, \beta-2+l}\left(B_{2^{3 / 2}}\right)}<\infty$. Hence, $\left(1-\bar{\eta}_{\infty}\right) v$ belongs to $E_{\beta}^{2}\left(d_{\vartheta}\right)$. In order to prove that $\bar{\eta}_{\infty} v$ belongs to $E_{\beta}^{2}\left(d_{\vartheta}\right)$ we apply Corollary 5.2. Thus, $v \in E_{\beta}^{2}\left(d_{\vartheta}\right)$. The functions $w$ and $\eta \varsigma_{k}$ vanish on the boundary of $d_{\vartheta}$, thus $v$ is a nonzero function from $\stackrel{\circ}{E}_{\beta}^{2}\left(d_{\vartheta}\right)$ which satisfies

$$
(\Delta-1) v=0 \text { in } d_{\vartheta}
$$


We recall that $v$ is smooth in $\dot{d}_{\vartheta}$. Now we set ${ }^{6}$

$$
u(x, z):=\mathcal{F}_{\xi \mapsto z}^{-1}\left[|\xi|^{\beta-1}(1+|\xi|)^{-1} v(|\xi| x)\right](z) \quad \text { for } \quad x \in \dot{d}_{\vartheta}, z \in \mathbb{R} .
$$

Therefore $0 \not \equiv u \in \stackrel{\circ}{H}_{\beta}^{2}\left(D_{\vartheta}\right)$ is well defined. Multiplying both sides of (39) by $|\xi|^{\beta+1}(1+$ $|\xi|)^{-1}$ we get $\left(|\xi|^{2} \Delta_{\eta}-|\xi|^{2}\right)\left[|\xi|^{\beta-1}(1+|\xi|)^{-1} v(\eta)\right]=0$ for $(\eta, \xi) \in d_{\vartheta} \times \mathbb{R}$. Now, substituting $x:=|\xi|^{-1} \eta$ we obtain $\left(\Delta_{x}-|\xi|^{2}\right)\left[|\xi|^{\beta-1}(1+|\xi|)^{-1} v(|\xi| x)\right]=0$ for $(x, \xi) \in d_{\vartheta} \times \mathbb{R}$. Therefore we have

$$
\left(\Delta_{x}-|\xi|^{2}\right) \hat{u}(x, \xi)=0 \quad \text { for } \quad x \in d_{\vartheta}, \quad \xi \in \mathbb{R},
$$

where the hat denotes the partial Fourier transform with respect to the $z$ variable. The function $u$ belongs to $\stackrel{\circ}{H}_{\beta}^{2}\left(D_{\vartheta}\right)$, thus $\Delta u \in L_{2, \beta}\left(D_{\vartheta}\right)$, hence using the Parseval identity and (41) we get $\Delta u=0$ in $D_{\vartheta}$, i.e. $\operatorname{ker} \mathcal{A}_{\beta}^{\vartheta} \neq\{0\}$.

Case of $1+k \frac{\pi}{\vartheta}+\mu \leq 0$. We choose a number $\gamma$ such that $0<1+k \frac{\pi}{\vartheta}+\gamma<2$. Then from the assumptions on $\mathcal{A}_{\mu}^{\vartheta}$, theorem 4.2 and proposition 5.7 we get that $\mathcal{A}_{\gamma}^{\vartheta}$ is onto $L_{2, \gamma}\left(D_{\vartheta}\right)$. Therefore, from the previous step we deduce $\operatorname{ker} \mathcal{A}_{\beta}^{\vartheta} \neq\{0\}$.

THEOREM 5.9. Assume that $\mu \in \mathbb{R}$ and $l \in \mathbb{N}$. Then, there exists a constant $c=c(\mu, l)$ with the following property: if $u \in L_{2, \mu}\left(D_{\vartheta}\right)$ is harmonic in $D_{\vartheta}$ and $u=0$ on $\Gamma_{k}$ for $k=0,1$, then

$$
\left\|D^{l} u\right\|_{L_{2, \mu+l}\left(D_{\vartheta}\right)} \leq c\|u\|_{L_{2, \mu}\left(D_{\vartheta}\right)} .
$$

In particular, if $u \in L_{2, \mu-2}\left(D_{\vartheta}\right)$ is harmonic in $D_{\vartheta}$ and $u=0$ on $\Gamma_{k}$ for $k=0,1$, then $u \in \stackrel{\circ}{H}_{\mu}^{2}\left(D_{\vartheta}\right)$.

Proof. We need the following notation:

$$
\begin{gathered}
\delta=\min \left\{\frac{\vartheta}{4}, \frac{\pi}{2}-\frac{\vartheta}{4}\right\}, \quad U_{\vartheta}=\left\{x \in \mathbb{R}^{3}:-\delta<\varphi<\vartheta+\delta, \quad r \in(0, \infty), \quad z \in \mathbb{R}\right\}, \\
U_{\vartheta}^{ \pm}=\left\{x \in \mathbb{R}^{3}: \frac{-\delta \pm \delta}{2}<\varphi<\vartheta+\frac{\delta \pm \delta}{2}, \quad r \in(0, \infty), \quad z \in \mathbb{R}\right\},
\end{gathered}
$$

where $(r, \varphi, z)$ are the cylindrical coordinates in $\mathbb{R}^{3}$. We define $\widetilde{u}$ as an extension of $u$ given in $U_{\vartheta}^{-}$(resp. $\left.U_{\vartheta}^{+}\right)$by the odd reflection of $u$ with respect to $\Gamma_{0}$ (resp. $\left.\Gamma_{1}\right)$. Then,

$$
\widetilde{u}_{\mid D_{\vartheta}} \equiv u, \quad\|\widetilde{u}\|_{L_{2, \mu}\left(U_{\vartheta}\right)} \leq 3\|u\|_{L_{2, \mu}\left(D_{\vartheta}\right)}, \quad \widetilde{u} \text { is harmonic in } U_{\vartheta} \text {. }
$$

First, we show that for some $c=c(\mu, l)$ the following estimate holds:

$$
\left\|D^{l} \widetilde{u}\right\|_{L_{2, \mu+l}\left(D_{\vartheta}\right)} \leq c\|\widetilde{u}\|_{L_{2, \mu}\left(U_{\vartheta}\right)} .
$$

For this purpose we define a suitable family of balls. For $n \in \mathbb{Z}$ we set $r_{n}:=\left(\frac{3}{8}\right)^{n}, R_{n}:=$ $\frac{\sin \delta}{2} r_{n}$. Let $K \in \mathbb{N}$ satisfy $K \geq \frac{\vartheta}{\arctan \frac{\sin \delta}{2}}$ and for $n, k \in \mathbb{Z}$ and $i \in I:=\{0,1, \ldots, K\}$

${ }^{6}$ This formula is well-defined for $v \in \stackrel{\circ}{\mathcal{D}}\left(\dot{d}_{\vartheta}\right)$ and proceeding similarly as in the proof of proposition 5.3 it can be shown that $\|v\|_{E_{\beta}^{2}\left(d_{\vartheta}\right)}=\sqrt{2}\|u\|_{H_{\beta}^{2}\left(D_{\vartheta}\right)}$. Furthermore, $u=0$ on $\Gamma_{k}$, because $v=0$ on $\gamma_{k}$. Therefore, formula (40) can be extended to all $v \in \stackrel{\circ}{E}_{\beta}^{2}\left(d_{\vartheta}\right)$. Then the 1.h.s. of (40) belongs to $\stackrel{\circ}{H}_{\beta}^{2}\left(D_{\vartheta}\right)$. 
we define points $P_{n, i, k}=\left(r_{n}, \frac{\vartheta}{K} i, \frac{\sin \delta}{2} k r_{n}\right)$ in cylindrical coordinates. Let $B_{n, i, k}^{1}$ (resp. $\left.B_{n, i, k}^{2}\right)$ denote the ball with center at $P_{n, i, k}$ and radius $R_{n}\left(2 R_{n}\right.$ resp.). The families $\left\{B_{n, i, k}^{1}\right\}_{n, k \in \mathbb{Z}, i \in I}$ and $\left\{B_{n, i, k}^{2}\right\}_{n, k \in \mathbb{Z}, i \in I}$ have the following properties:

$$
\begin{gathered}
D_{\vartheta} \subseteq \bigcup_{n, k \in \mathbb{Z}} \bigcup_{i \in I} B_{n, i, k}^{1} \subseteq \bigcup_{n, k \in \mathbb{Z}} \bigcup_{i \in I} B_{n, i, k}^{2} \subseteq U_{\vartheta}, \\
\quad \operatorname{diam} B_{n, i, k}^{1}=c(\delta) \operatorname{dist}\left(B_{n, i, k}^{1}, M\right), \\
\text { the order of the covering } \bigcup_{n, k \in \mathbb{Z}} \bigcup_{i \in I} B_{n, i, k}^{2} \text { is finite, }
\end{gathered}
$$

i.e. there exists a constant $N_{0}$ such that any intersection of $N_{0}+1$ elements from the family $\left\{B_{n, i, k}^{2}\right\}_{n, k \in \mathbb{Z}, i \in I}$ is empty. The function $\widetilde{u}$ is harmonic in each ball $B_{n, i, k}^{2}$, thus applying theorem 8.2 of [1] we obtain a universal constant $c_{0}=c_{0}(l)$ such that

$$
\sup _{B_{n, i, k}^{1}}\left|D^{l} \widetilde{u}\right| \leq c_{0} R_{n}^{-l-\frac{3}{2}}\|\widetilde{u}\|_{L_{2}\left(B_{n, i, k}^{2}\right)} .
$$

Thus, using the Hölder inequality and (49) we get

$$
\begin{aligned}
\left\|D^{l} \widetilde{u}\right\|_{L_{2, \mu+l}\left(B_{n, i, k}^{1}\right)} & \leq\left|B_{n, i, k}^{1}\right|^{1 / 2} \cdot \sup _{B_{n, i, k}^{1}} r^{\mu+l} \cdot \sup _{B_{n, i, k}^{1}}\left|D^{l} \widetilde{u}\right|^{2} \\
& \leq(4 / 3 \pi)^{1 / 2} c_{0} R_{n}^{-l} \cdot \sup _{B_{n, i, k}^{1}} r^{\mu+l} \cdot\|\widetilde{u}\|_{L_{2}\left(B_{n, i, k}^{2}\right)} \\
& \leq(4 / 3 \pi)^{1 / 2} c_{0} R_{n}^{-l} \cdot \sup _{B_{n, i, k}^{1}} r^{\mu+l} \cdot \sup _{B_{n, i, k}^{2}} r^{-\mu} \cdot\|\widetilde{u}\|_{L_{2, \mu}\left(B_{n, i, k}^{2}\right)} .
\end{aligned}
$$

By direct calculations we verify that $R_{n}^{-l} \sup _{B_{n, i, k}^{1}} r^{\mu+l} \cdot \sup _{B_{n, i, k}^{2}} r^{-\mu} \leq c$, where $c$ depends only on $\mu$ and $l$, thus we have

$$
\left\|D^{l} \widetilde{u}\right\|_{L_{2, \mu+l}\left(B_{n, i, k}^{1}\right)} \leq c(\mu, l)\|\widetilde{u}\|_{L_{2, \mu}\left(B_{n, i, k}^{2}\right)} .
$$

Therefore, utilizing (46) and (48) we get (45). Finally, using (44) we get the desired inequality.

THEOREM 5.10. Assume that $\mu$ is real. Then, the transformation $w \mapsto w \cdot r^{-2 \mu^{\prime}} \equiv w^{\prime}$ is an isometrical isomorphism from $L_{2, \mu-2}\left(D_{\vartheta}\right)$ onto $L_{2, \mu^{\prime}}\left(D_{\vartheta}\right)$ such that $w \in \mathcal{K}_{\mu}^{\vartheta} \Leftrightarrow w^{\prime} \in \mathcal{N}_{\mu^{\prime}}^{\vartheta}$. In particular, $\operatorname{dim} \mathcal{K}_{\mu}^{\vartheta}=\operatorname{dim} \mathcal{N}_{\mu^{\prime}}^{\vartheta}$

Proof. First, we notice that the above transformation is an isometry from $L_{2, \mu-2}\left(D_{\vartheta}\right)$ onto $L_{2, \mu^{\prime}}\left(D_{\vartheta}\right)$. Indeed, because of $\mu^{\prime}=2-\mu$ we have $\|w\|_{L_{2, \mu-2}\left(D_{\vartheta}\right)}=\left\|w^{\prime}\right\|_{L_{2, \mu^{\prime}}\left(D_{\vartheta}\right)}$. Now, assume that $w \in \mathcal{K}_{\mu}^{\vartheta}$. Then $w \in \stackrel{\circ}{H}_{\mu}^{2}\left(D_{\vartheta}\right)$ and $\Delta w=0$ in $D_{\vartheta}$. For $u \in \stackrel{\circ}{\mathcal{D}}\left(\dot{\circ}_{\vartheta}\right)$ we have $w \in H^{2}(\operatorname{supp} u)$ and the Green formula holds for $u$ and $w$, i.e.

$$
\int_{D_{\vartheta}} w \cdot \Delta u d x-\int_{D_{\vartheta}} \Delta w \cdot u d x=\sum_{k=0}^{1} \int_{\Gamma_{k}} w \frac{\partial u}{\partial n} d \sigma-\int_{\Gamma_{k}} \frac{\partial w}{\partial n} u d \sigma .
$$

The function $w$ is harmonic in $D_{\vartheta}$ and $w, u$ vanish on $\Gamma_{k}$, hence we get $0=\int_{D_{\vartheta}} w \cdot \Delta u d x=$ $\int_{D_{\vartheta}} w^{\prime} \cdot \Delta u \cdot r^{2 \mu^{\prime}} d x$. The function $w^{\prime}$ belongs to $L_{2, \mu^{\prime}}\left(D_{\vartheta}\right)$ and $\stackrel{\circ}{\mathcal{D}}\left(\stackrel{\bullet}{D}_{\vartheta}\right)$ is dense in $\stackrel{\circ}{H}_{\mu^{\prime}}^{2}\left(D_{\vartheta}\right)$, 
thus we get

$$
\int_{D_{\vartheta}} w^{\prime} \cdot \Delta u \cdot r^{2 \mu^{\prime}} d x=0 \text { for } u \in \stackrel{\circ}{H}_{\mu^{\prime}}^{2}\left(D_{\vartheta}\right) .
$$

By the definition this means that $w^{\prime} \in \mathcal{N}_{\mu^{\prime}}^{\vartheta}$. Now, suppose that $w^{\prime} \in \mathcal{N}_{\mu^{\prime}}^{\vartheta}$. Then $w=$ $w^{\prime} \cdot r^{2 \mu^{\prime}}$ is harmonic in $D_{\vartheta}$. If $K \subseteq \dot{D}_{\vartheta}$ is compact with smooth boundary, then $w \in L^{2}(K)$ and $\Delta w \in L^{2}(K)$. Therefore, the function $w$ has the traces $w_{\mid \partial K}$ and $\frac{\partial w}{\partial n}{ }_{\mid \partial K}$, which are well defined functionals on $H^{\frac{1}{2}}(\partial K)$ and $H^{\frac{3}{2}}(\partial K)$ respectively and the Green formula holds:

$$
\int_{K} w \cdot \Delta u d x-\int_{K} \Delta w \cdot u d x=\langle w, \partial u / \partial n\rangle_{L^{2}(\partial K)}-\langle\partial w / \partial n, u\rangle_{L^{2}(\partial K)},
$$

where $u \in H^{2}(K)$. Let $\varphi_{k}$ be smooth with the compact support in $\Gamma_{k}, k=0,1$. From the trace theorem we obtain $u \in H^{2}\left(D_{\vartheta}\right)$ such that $u_{\mid \Gamma_{k}}=0,\left.\quad \frac{\partial u}{\partial n}\right|_{k}=\varphi_{k}, k=0,1$. We may assume that $\operatorname{supp} u \subseteq K \subseteq \dot{D}_{\vartheta}$, where $K$ is compact and $u=0$ and $\frac{\partial u}{\partial n}=0$ on $\partial K \backslash\left(\Gamma_{0} \cup \Gamma_{1}\right)$. We put such $u$ in (51). On the other hand $\int_{K} w \cdot \Delta u d x=\int_{D_{\vartheta}} w \cdot \Delta u d x=0$, because $u$ belongs to $\stackrel{\circ}{H}_{\mu}^{2}\left(D_{\vartheta}\right)$ and we use (50). Therefore (51) reduces to the equality $0=\sum_{k=0}^{1}\left\langle w, \varphi_{k}\right\rangle_{L^{2}\left(\Gamma_{k}\right)}$. The functions $\varphi_{k}$ were arbitrary and smooth with compact supports in $\Gamma_{k}$, thus we deduce that $w_{\mid \Gamma_{k} \cap K}=0$ for each compact set $K \subseteq \dot{D}_{\vartheta}$. The function $w$ is harmonic in $D_{\vartheta}$, hence it is smooth in $D_{\vartheta}$. If we extend $w$ to the set $U_{\vartheta}$ (see (43)) by the odd reflection with respect to $\Gamma_{k}$ (analogously as in the proof of theorem 5.9), then the extension $\widetilde{w}$ in harmonic in $U_{\vartheta}$, thus $\widetilde{w}$ in smooth in $U_{\vartheta}$, hence $\widetilde{w}_{\mid D_{\vartheta}}=w$ is smooth in $\dot{D}_{\vartheta}$, i.e. smooth up to the boundary. Therefore, $w \in \stackrel{\circ}{C}^{\infty}\left(\dot{d}_{\vartheta}\right)$ and $w \in L_{2, \mu-2}\left(D_{\vartheta}\right)$, hence the assumptions of Theorem 5.9 are satisfied. Then, we deduce that $w \in \stackrel{\circ}{H}_{\mu}^{2}\left(D_{\vartheta}\right)$ and $\Delta w=0$ in $D_{\vartheta}$. By the definition this means that $w \in \mathcal{K}_{\mu}^{\vartheta}$.

REMARK 5.11. Now, we can give another proof of theorem 3.7, which is based on the above theorem. Indeed, first we assume that $u \in \stackrel{\circ}{\mathcal{D}}\left(\dot{\bullet}_{\vartheta}\right)$ and using the Poincaré inequality and then integrating by parts we get

$$
\|u\|_{L_{2,-1}\left(D_{\vartheta}\right)}^{2} \leq(\vartheta / \pi)^{2}\|\nabla u\|_{L_{2,0}\left(D_{\vartheta}\right)}^{2} \leq \frac{1}{2}(\vartheta / \pi)^{4}\|\Delta u\|_{L_{2,1}\left(D_{\vartheta}\right)}^{2}+\frac{1}{2}\|u\|_{L_{2,-1}\left(D_{\vartheta}\right)}^{2} .
$$

Therefore, with the help of lemma 3.5 we deduce that $\mathcal{K}_{1}^{\vartheta}=\{0\}$ and the range of $\mathcal{A}_{1}^{\vartheta}$ is closed in $L_{2,1}\left(D_{\vartheta}\right)$. On the other hand, by using theorem 5.10 we get $\operatorname{dim} \mathcal{N}_{1}^{\vartheta}=\operatorname{dim} \mathcal{K}_{1}^{\vartheta}$, hence $\mathcal{N}_{1}^{\vartheta}=\{0\}$. Therefore $\mathcal{A}_{1}^{\vartheta}$ is an isomorphism and utilizing proposition 5.7 we deduce that $\mathcal{A}_{\mu}^{\vartheta}$ is an isomorphism for $\mu \in(1-\pi / \vartheta, 1+\pi / \vartheta)$.

THEOREM 5.12. Assume that $\mu>1+\frac{\pi}{\vartheta}$ and $\mu \neq 1+\pi \frac{k}{\vartheta}$ for $k=1,2, \ldots$ Then $\mathcal{K}_{\mu}^{\vartheta} \neq\{0\}$.

Proof. Let us suppose $\mathcal{K}_{\mu}^{\vartheta}=\{0\}$. Then, from theorem 5.10 we get $\mathcal{N}_{\mu^{\prime}}^{\vartheta}=\{0\}$, i.e. the range of $\mathcal{A}_{\mu^{\prime}}^{\vartheta}$ is dense in $L_{2, \mu^{\prime}}\left(D_{\vartheta}\right)$. We notice that $\mu^{\prime}<1-\frac{\pi}{\vartheta}$ and $\mu^{\prime} \neq 1-\pi \frac{k}{\vartheta}$ for $k=1,2, \ldots$. Therefore $\mu^{\prime}$ satisfies the assumptions of theorem 4.2 , hence we deduce that the range of $\mathcal{A}_{\mu^{\prime}}^{\vartheta}$ is a closed subspace of $L_{2, \mu^{\prime}}\left(D_{\vartheta}\right)$. Thus $\mathcal{A}_{\mu^{\prime}}^{\vartheta}$ is onto $L_{2, \mu^{\prime}}\left(D_{\vartheta}\right)$. Applying theorem 5.8 with $\mu^{\prime}$ and $\beta=1$ we obtain that $\mathcal{A}_{1}^{\vartheta}$ is not injective, contrary to theorem 3.7 . 
Proposition 5.13. Assume that $\mu$ is real and $\mathcal{K}_{\mu}^{\vartheta} \neq\{0\}$. Then $\operatorname{dim} \mathcal{K}_{\mu}^{\vartheta}=\infty$.

Proof. If $0 \not \equiv v \in \mathcal{K}_{\mu}^{\vartheta}$, then the functions $v^{k}(x, z)=v(x, z-k)$ for $k \in \mathbb{Z}$ span an infinite dimensional subspace of $\mathcal{K}_{\mu}^{\vartheta}$.

From Theorems 4.2, 5.10, 5.12 and the above proposition we get:

Corollary 5.14. Assume that $\mu \neq 1-\pi \frac{k}{\vartheta}$ for $k \in \mathbb{Z} \backslash\{0\}$. If $\mu<1-\frac{\pi}{\vartheta}$, then $\operatorname{dim} \mathcal{K}_{\mu}^{\vartheta}=0$ and $\operatorname{dim} \mathcal{N}_{\mu}^{\vartheta}=\infty$. If $\mu>1+\frac{\pi}{\vartheta}$, then $\operatorname{dim} \mathcal{K}_{\mu}^{\vartheta}=\infty$ and $\operatorname{dim} \mathcal{N}_{\mu}^{\vartheta}=0$.

Therefore, theorem 2.1 is a consequence of theorems 3.7, 4.2 and corollary 5.14.

6. The parabolic problem. In this section we give a proof of theorem 2.2. First, we notice that from theorems 2.3.1 and 2.3.5 of [10] we get a priori estimates for the solution of the Poisson equation in $\stackrel{\circ}{H}_{\mu}^{2}\left(d_{\vartheta, 1}\right)$ i.e. if $\mu \neq 1-k \frac{\pi}{\vartheta}$ for $k=1,2, \ldots$, and $\mu<1+\frac{\pi}{\vartheta}$, then

$$
\|u\|_{H_{\mu}^{2}\left(d_{\vartheta, 1}\right)} \leq c\|\Delta u\|_{L_{2, \mu}\left(d_{\vartheta, 1}\right)} \quad \text { for } \quad u \in \stackrel{\circ}{H_{\mu}^{2}}\left(d_{\vartheta, 1}\right)
$$

where $c=c(\mu, \vartheta)$. Thus, we get:

TheOREM 6.1. Assume that $\mu \neq 1-k \frac{\pi}{\vartheta}$ for $k=1,2, \ldots, \mu<1$ and $T>0$. Then there exist constants $c_{1}, c_{2}$ such that

$$
\begin{gathered}
\|u\|_{H_{\mu}^{2}\left(D_{\vartheta, 1}\right)} \leq c_{1}(\mu, \vartheta)\|\Delta u\|_{L_{2, \mu}\left(d_{\vartheta, 1}\right)} \quad \text { for } \quad u \in \stackrel{\circ}{H}_{\mu}^{2}\left(D_{\vartheta, 1}\right), \\
\|u\|_{H_{\mu}^{2,1}\left(D_{\vartheta, 1}^{T}\right)} \leq c_{2}(\mu, \vartheta, T)\left\|u_{t}-\Delta u\right\|_{L_{2, \mu}\left(D_{\vartheta, 1}^{T}\right)} \quad \text { for } u \in{\stackrel{\circ}{H_{\circ}}}_{\mu}^{2,1}\left(D_{\vartheta, 1}^{T}\right),
\end{gathered}
$$

Proof. We proceed as in the proof of theorem 4.2, but first we have to extend periodically functions from $d_{\vartheta, 1} \times(-1,1)$ to $d_{\vartheta, 1} \times T$, where $T$ is the one dimensional torus. In the next step, we repeat the proof of lemma 4.4 utilizing estimate (52).

Now we define a bilinear form $B_{\mu}[\cdot, \cdot]$ on $\stackrel{\circ}{H}_{\mu-1}^{1}\left(D_{\vartheta, 1}\right)$ by the formula

$$
B_{\mu}[u, \phi]=\int_{D_{\vartheta, 1}} \nabla u \cdot \nabla \bar{\phi} \cdot r^{2 \mu-2} d x+(2 \mu-2) \int_{D_{\vartheta, 1}} \nabla u \cdot \nabla r \cdot \bar{\phi} r^{2 \mu-3} d x .
$$

It is clear that $B_{\mu}[\cdot, \cdot]$ is continuous on $\stackrel{\circ}{H}_{\mu-1}^{1}\left(D_{\vartheta, 1}\right)$. As in the proof of theorem 3.7 , we show that

$$
\text { if }|\mu-1|<\frac{\pi}{\vartheta} \text {, then } B_{\mu}[\cdot, \cdot] \text { is elliptic on } \stackrel{\circ}{H_{\mu-1}^{1}}\left(D_{\vartheta, 1}\right) .
$$

Therefore, using the Lax-Milgram theorem and lemma 3.5 we get

Corollary 6.2. Assume that $\mu \in\left(1-\frac{\pi}{\vartheta}, 1+\frac{\pi}{\vartheta}\right)$. Then for each $f \in L_{2, \mu}\left(D_{\vartheta, 1}\right)$ there exists a unique $u \in \stackrel{\circ}{H}_{\mu}^{2}\left(D_{\vartheta, 1}\right)$ which satisfies $\Delta u=f$ in $D_{\vartheta, 1}$ and $\|u\|_{H_{\mu}^{2}\left(D_{\vartheta, 1}\right)} \leq$ $c(\mu, \vartheta)\|f\|_{L_{2, \mu}\left(D_{\vartheta, 1}\right)}$.

Now, we shall show existence of weak solutions of the parabolic problem $(\mathrm{P})$. We need the following notation:

$$
V_{\mu}=\left\{u \in L^{2}\left(0, T: \stackrel{\circ}{H}_{\mu-1}^{1}\left(D_{\vartheta, 1}\right)\right): \underset{t \in[0, T]}{\left.\operatorname{ess} \sup _{t}\|u\|_{L_{2, \mu-1}\left(D_{\vartheta, 1}\right)}<\infty\right\}},\right.
$$




$$
P_{\mu}=\left\{\psi \in L^{2}\left(0, T: \stackrel{\circ}{H}_{\mu-1}^{1}\left(D_{\vartheta, 1}\right)\right): \frac{d}{d t} \psi \in L^{2}\left(0, T ; L_{2, \mu}\left(D_{\vartheta, 1}\right)\right), \quad \psi(\cdot, T)=0\right\} .
$$

Definition 6.3. The function $u \in V_{\mu}$ is a weak solution of the problem (P) if

$$
\int_{D_{\vartheta, 1}} u \psi r^{2 \mu-2} d x-\int_{0}^{t} \int_{D_{\vartheta, 1}} u \psi_{t} r^{2 \mu-2} d x d t+\int_{0}^{t} B[u, \psi] d t=\int_{0}^{t} \int_{D_{\vartheta, 1}} f \psi r^{2 \mu-2} d x d t
$$

for a.e. $t \in(0, T)$ and $\psi \in P_{\mu}$.

We shall show that if $|\mu-1|<\frac{\pi}{\vartheta}$, then there exists a weak solution of the problem (P). For this purpose we apply the Galerkin method, but first we have to define a fundamental system $\left\{\varphi_{n}\right\}_{n \in \mathbb{N}}$ in $L_{2, \mu-1}\left(D_{\vartheta, 1}\right)$. In order to do it, we set $\widetilde{B}[u, \varphi]=\frac{1}{2}(B[u, \varphi]+B[\varphi, u])$, thus $\widetilde{B}[\cdot, \cdot]$ is a bilinear, symmetric, continuous and elliptic form on $\stackrel{\circ}{H}_{\mu-1}^{1}\left(D_{\vartheta, 1}\right)$. We define the operator $\mathbb{T}: L_{2, \mu-1}\left(D_{\vartheta, 1}\right) \rightarrow \stackrel{\circ}{H_{\mu-1}^{1}}\left(D_{\vartheta, 1}\right)$ by the formula

$$
\mathbb{T} f=u \Leftrightarrow \widetilde{B}[u, \varphi]=\int_{D_{\vartheta, 1}} f \varphi r^{2 \mu-2} d x \quad \text { for } \varphi \in \stackrel{\circ}{H}_{\mu-1}^{1}\left(D_{\vartheta, 1}\right) .
$$

It is linear, continuous, self-adjoint and compact in $L_{2, \mu-1}\left(D_{\vartheta, 1}\right)$, since the imbedding $\stackrel{\circ}{H}_{\mu-1}^{1}\left(D_{\vartheta, 1}\right) \subset L_{2, \mu-1}\left(D_{\vartheta, 1}\right)$ is compact. Therefore the set $\left\{\varphi_{n}\right\}$ of eigenfunctions of $\mathbb{T}$ forms a fundamental system in $L_{2, \mu-1}\left(D_{\vartheta, 1}\right)$, which has the additional property $\left\{\varphi_{n}\right\} \subseteq$ $\stackrel{\circ}{H}_{\mu-1}^{1}\left(D_{\vartheta, 1}\right)$. Furthermore, we may assume that $\left\langle\varphi_{n}, \varphi_{k}\right\rangle_{L_{2, \mu-1}\left(D_{\vartheta, 1}\right)}=\delta_{n k}$.

We find the approximate solution of $(\mathrm{P})$ in the form $u^{N}(x, t)=\sum_{n=1}^{N} c_{n}^{N}(t) \cdot \varphi_{n}(x)$, where $c_{n}^{N}(t)$ are solutions of an appropriate system of ordinary differential equations. Next, we obtain the estimate $\left\|u^{N}\right\|_{V_{\mu}} \leq c(\mu, \vartheta)\|f\|_{L_{2, \mu}\left(D_{\vartheta, 1}^{T}\right)}$ and then using the standard argument we conclude that there exists a weak solution $u \in V_{\mu}$ of $(\mathrm{P})$ and

$$
\|u\|_{V_{\mu}} \leq c(\mu, \vartheta)\|f\|_{L_{2, \mu}\left(D_{\vartheta, 1}^{T}\right)}
$$

Further, utilizing the regularity argument we show that $u \in H^{2,1}\left(D_{\vartheta, 1}^{T} \cap\{(x, t): r>\varepsilon\}\right)$ for $\varepsilon>0$. Finally, proceeding as in the proof of lemma 3.5 we get the estimate

$$
\|u\|_{H_{\mu}^{2,1}\left(D_{\vartheta, 1}^{T}\right)} \leq c(\mu)\left(\|f\|_{L_{2, \mu}\left(D_{\vartheta, 1}^{T}\right)}+\|u\|_{L^{2}\left(0, T ; H_{\mu-1}^{1}\left(D_{\vartheta, 1}\right)\right)}\right) .
$$

Therefore, (57) and (58) give us the desired inequality and the proof of theorem 2.2 is finished.

REMARK 6.4. The problem (P) is called the model problem and may be used in solving other parabolic problems with variable coefficients in domains with edges. This may be done as an application of the regularizer technique (see, for example [8]).

Acknowledgements. I would like to thank to Professor Piotr Rybka for his important suggestions and the assistance during the preparation of this paper. I am extremely grateful to him for his encouragement for this work. I would like to thank the reviewer for his valuable remarks, which made this paper essentially improved.

The research is partially supported by MNiSW grants no 1 P03A 02130 and N N201 268935. 


\section{References}

[1] S. Axler, P. Bourdon and W. Ramey, Harmonic Function Theory, Springer-Verlag, New York, 2001.

[2] G. H. Hardy, J. E. Littlewood and G. Pólya, Inequalities, Cambridge University Press, 1934.

[3] V. A. Kondrat'ev, Boundary value problems for elliptic equations in domains with conical or angular points, Trudy Moskov. Mat. Obšč. 16 (1967).

[4] A. Kubica, The regulatity of weak and very weak solutions of the Poisson equation on polygonal domains with mixed boundary conditions (part II), Appl. Math. (Warsaw) 32 (2005), 17-36.

[5] A. Kubica and W. Zajączkowski, A priori estimates in weighted space for solutions of the Poisson and the heat equations, Appl. Math. (Warsaw) 34 (2007), 431-444.

[6] A. Kufner and J. Rákosník, Linear elliptic boundary value problems and weighted Sobolev spaces: a modified approach, Math. Slovaca 34 (1984), 185-197.

[7] A. Kufner and A. M. Sändig, Some Applications of Weighted Sobolev Spaces, TeubnerTexte zur Mathematik 100, Teubner, Leipzig.

[8] O. A. Ladyženskaja, V. A. Solonnikov and N. N. Uralceva, Linear and Quasilinear Equations of Parabolic Type, "Nauka", Moscow, 1967 (in Russian); English transl., Amer. Math. Soc., Providence, RI, 1968.

[9] V. Maz'ya, S. Nazarov and B. Plamenevsky, Asymptotic Theory of Elliptic Boundary Value Problems in Singularly Perturbed Domains I, II, Operator Theory: Advances and Applications 111, 112, Birkhäuser, Basel, 2000.

[10] S. Nazarov and B. Plamenevsky, Elliptic Problems in Domains with Piecewise Smooth Boundaries, Walter de Gruyter, Berlin, 1994. 